\title{
Formation of hollow carbon nanoshells from thiol stabilised silver nanoparticles via heat treatment
}

Julie A Watts ${ }^{1,2}$, Mike W Fay ${ }^{3}$, Graham A Rance ${ }^{3}$, Paul D Brown*1,3, and Andrei N Khlobystov ${ }^{2,3}$

${ }^{1}$ Department of Mechanical, Materials and Manufacturing Engineering, Faculty of Engineering, University of Nottingham, University Park, Nottingham, NG7 2RD.

${ }^{2}$ School of Chemistry, University of Nottingham, University Park, Nottingham, NG7 2RD. ${ }^{3}$ Nanoscale and Microscale Research Centre, University of Nottingham, University Park, Nottingham, NG7 2RD.

\begin{abstract}
Uniform, < $10 \mathrm{~nm}$ sized, hollow carbon nano-shells (HCNS) have been prepared via a single-step, thermal treatment of alkanethiol stabilised Ag nanoparticles (TS-AgNP). Direct evidence for the formation of spherical HCNS from TS-AgNP is provided by in situ MEMS heating on $\mathrm{Si}_{3} \mathrm{~N}_{4}$ supports within a TEM, and ex situ thermal processing of TS-AgNP on carbon nanotube supports. A mechanism is proposed for the thermally driven, templated formation of HCNS from the TS-AgNP stabilising layer, with Ag catalysing the graphitisation of carbon in advance of thermally induced AgNP template removal. This facile processing route provides for excellent size control of the HCNS product via appropriate AgNP template selection. However, a rapid rate of heating was found to be crucial for the formation of well-defined HCNS, whilst a slow heating rate gave a much more disrupted product, comprising predominantly lacy carbon with decreased levels of graphitic ordering, reflecting a competition between the thermal transformation of the TS-layer and the rate of removal of the AgNP template.
\end{abstract}

\section{Introduction}

Carbon is abundant, non-toxic and forms a diverse range of allotropes on the nanoscale, including graphene, nanofibres, nanotubes, quantum dots and fullerenes [1]. In particular, hollow, nanostructured carbons provide for a wide variety of applications including catalysis [2], gas-storage [3] and for drug delivery systems [4]. However, fullerenes have limited volumes for use as containers, whilst quasi one dimensional (1D) carbon nanotubes (CNT) and nanofibers (NF) with larger volumes have limited solubilities and tend to bundle [5].

* Corresponding author Tel: 0115951 3748. E-mail: paul.brown@nottingham.ac.uk 
Hollow carbon nanoshell (HCNS) structures, of size $\sim 200 \mathrm{~nm}$, were documented originally as by-products from the carbon-arc processing of CNT [6]. More recently, HCNS structures with sizes ranging from $\sim 70-800 \mathrm{~nm}$ have been produced using a variety of sacrificial templating and annealing processes [7-10]. The generic approach is that of hydrocarbon templating of mesoporous shell $\mathrm{SiO}_{2}$ nanoparticles (NP), [11], thermal processing (at $\sim 450-1000{ }^{\circ} \mathrm{C}$ in an inert atmosphere) to create graphitic $\mathrm{C}$, followed by template removal (using dilute $\mathrm{HF}$ or $\mathrm{NaOH}$ ) to create bespoke HCNS structures. As part of this processing route, it is noted that a processing temperature of $\sim 600^{\circ} \mathrm{C}$ is considered necessary for the effective graphitisation of amorphous carbon [12]. Alternative strategies for creating HCNS structures $(50-150 \mathrm{~nm})$ include soft-templating, involving micelles or droplets [13], or template-free methods of production [14], however, these still present significant challenges, e.g. a lack of scalability [15].

Here, we demonstrate the novel use of thiol-stabilised Ag nanoparticles (TS-AgNP) to synthesise hollow carbon nanoshell (HCNS) structures of $<10 \mathrm{~nm}$ size. This self-contained process may be performed under vacuum, with the thiol stabilising layer providing the source of carbon and the AgNP template dictating the morphology of the resultant carbon shell, whilst thermal processing at $\sim 600-900^{\circ} \mathrm{C}$ catalyses HCNS production, in advance of thermally induced template removal.

\section{Experimental}

Silver nanoparticles (AgNP) stabilised with 1-dodecanethiol (DDM) were synthesised via a modified Brust-Schiffrin reduction [16] as follows: To a solution of silver nitrate $(0.3 \mathrm{mmol} /$ $30 \mathrm{~mL}$ ethanol), DDM (0.37 mmol) was added slowly with vigorous stirring for 15 minutes. This was followed by dropwise addition of excess sodium borohydride (saturated / $60 \mathrm{~mL}$ ethanol) and the solution stirred vigorously for $2 \mathrm{~h}$. The product was then precipitated through the addition of $250 \mathrm{~mL}$ ethanol and refrigeration at $-15^{\circ} \mathrm{C}$ for $24 \mathrm{~h}$. The TS-AgNP product was isolated by vacuum filtration using a $0.2 \mu \mathrm{m}$ PTFE membrane, washed with $10 \mathrm{~mL}$ toluene, followed by washing with $200 \mathrm{~mL}$ acetone and drying under vacuum. All reagents were used as purchased from Sigma-Aldrich UK without further purification and syntheses were performed at room temperature (RT) using standard glassware and laboratory equipment. TSAgNP samples were processed thermally either in situ within a TEM on a micro-electro- 
mechanical systems (MEMS) chip or ex situ within a furnace on multi-walled carbon nanotube (MWCNT) supports.

Samples for in situ TEM investigation were dissolved in $\mathrm{CHCl}_{3}(0.5 \mathrm{mg} / \mathrm{mL})$ and drop-cast onto a MEMS-based EMheaterchip ${ }^{\mathrm{TM}}\left(\mathrm{Si}_{3} \mathrm{~N}_{4}\right.$ support film; DENSolutions), with drying at RT. TEM with a heating holder (DENSolutions; SH30) was performed using a JEOL 2100 Plus with a $\mathrm{LaB}_{6}$ source operating at $80 \mathrm{kV}$, with a Gatan Ultrascan $1000 \mathrm{XP}$ camera, or on a JEOL $2100 \mathrm{~F}$ operating at $100 \mathrm{kV}$, with a Gatan Orius SC1000 camera; with Oxford Instruments energy dispersive X-ray spectrometers (X-Max 100 TLE, 2100 Plus and X-Max 80T, 2100F, respectively). Two different heating protocols were developed to elucidate the effect of heating rate on the formation of HCNS structures. Firstly, rapid heating from RT $\left(23^{\circ} \mathrm{C}\right)$ to $850^{\circ} \mathrm{C}$ (at $92^{\circ} \mathrm{C} / \mathrm{s}$ ), followed by a hold time of 80 minutes, then cool down to RT within $30 \mathrm{~s}$ (Thermal Protocol 1 (TP-1)). Secondly, slow heating from RT to $650^{\circ} \mathrm{C}$ (at $1.5^{\circ} \mathrm{C} / \mathrm{s}$ ), followed by a hold time of 2 minutes, then slow heating to $900^{\circ} \mathrm{C}$ (at $2^{\circ} \mathrm{C} / \mathrm{s}$ ) and a further hold time of 15 minutes, followed by cool down to RT within $30 \mathrm{~s}$ (Thermal Protocol 2 (TP-2)). TS-AgNP and resultant HCNS sizes and size distributions were determined from TEM micrographs using ImageJ 1.48v [17], Java 1.6.0-20 and OriginPro 8 software.

Micro Raman spectroscopy was performed using a Horiba Jobin Yvon LabRAM HR Raman spectrometer. Spectra were acquired using a $532 \mathrm{~nm}$ laser at $0.3 \mathrm{~mW}$ power, to avoid local heating of the sample, a 100x objective lens and a $50 \mu \mathrm{m}$ confocal pinhole. The spectral resolution in this configuration is better than $\sim 1.6 \mathrm{~cm}^{-1}$. To simultaneously scan a range of Raman shifts, a 600 lines $\mathrm{mm}^{-1}$ rotatable diffraction grating along a path length of $800 \mathrm{~mm}$ was employed. Spectra were acquired using a Synapse CCD detector (1024 pixels), thermoelectrically cooled to $-60^{\circ} \mathrm{C}$. Before the collection of spectra, the instrument was calibrated using the zero-order line and a standard $\mathrm{Si}(100)$ reference band at $520.7 \mathrm{~cm}^{-1}$. Point spectra were acquired over the range $800-2000 \mathrm{~cm}^{-1}$ with an acquisition time of $60 \mathrm{~s}$ and 16 accumulations to improve signal to noise ratio. The DuoScan ${ }^{\mathrm{TM}}$ functionality was employed to confer an effective laser spot size of $20 \times 5 \times 4 \mu \mathrm{m}$ in the xyz directions, respectively. As such, the obtained spectra represent an average of all HCNS structures (produced in situ) present within a TEM grid window. Spectra were baseline-corrected using a third-order polynomial subtraction and fit with a five-band model analogous to that described by Sadezky et al. [18] using Labspec 6.4.3 software. The G and D2 bands are described by Lorenzian peak shapes, whereas the D1, D3 and D4 bands are Gaussian. 
Samples for ex situ thermal processing were prepared by mixing TS-AgNP (3.6 mg in $1.5 \mathrm{ml}$ cyclohexane) with MWCNT (3.9 mg; Nanothinx S. A.), via brief sonication then shaking for $15 \mathrm{~min}$. After isolation by filtration $(0.2 \mu \mathrm{m}$ PTFE membrane) and washing with cyclohexane, ethanol and acetone $(10 \mathrm{~mL})$, the resultant solid (TS-AgNP@CNT) was dried for $16 \mathrm{~h}$. The TS-AgNP@CNT (3 mg) was then sealed inside a quartz ampoule under vacuum $\left(10^{-5} \mathrm{mbar}\right)$ and placed inside a pre-heated furnace at $850^{\circ} \mathrm{C}$ for 180 minutes (Nabertherm $\mathrm{GmbH}$ P330 muffle furnace), before cooling naturally, outside the furnace, to RT. As-prepared TSAgNP@CNT samples and ex situ thermally treated products were dissolved in cyclohexane $(0.5 \mathrm{mg} / \mathrm{mL})$ and drop-cast onto TEM grids (holey or lacey carbon films on 300 or 200 mesh $\mathrm{Cu}$; Agar Scientific Ltd). In this case, TEM was performed using a JEOL 2100 Plus operating at $200 \mathrm{kV}$, or JEOL $2100 \mathrm{~F}$ operating at $100 \mathrm{kV}$ or $200 \mathrm{kV}$, with either single tilt (JEOL 21010) or high tilt (GATAN 916) holders to facilitate tilt series imaging of the resultant HCNS structures edge-on to the support. To measure layer spacings of HCNS, images were calibrated against the graphitic spacing within MWCNT (0.34 nm [19]), as an internal reference.

Complementary Fourier transform infrared spectroscopy (FTIR) of the starting TS-AgNP was performed using a Bruker Tensor 27; $0.5 \mathrm{mg}$ of solid sample was ground with $100 \mathrm{mg} \mathrm{KBr}$ and pressed to form a disc, whilst for Nuclear Magnetic Resonance (NMR) samples were prepared by dissolution of $\mathrm{TS}-\mathrm{AgNP}$ in $\mathrm{CDCl}_{3}(2 \mathrm{mg} / 4.0 \mathrm{ml})$ and filtering, prior to data collection using a Bruker AV(III) $500 \mathrm{MHz}$.

\section{Results}

As-synthesised TS-AgNP samples were characterised initially using TEM and EDS to evaluate their starting morphologies prior to thermal processing. Figure 1a presents a bright field, diffraction contrast TEM image of TS-AgNP dispersed onto the planar $\mathrm{Si}_{3} \mathrm{~N}_{4}$ support of a MEMs heating chip, recorded at RT. In this case, the imaging conditions revealed the crystalline AgNP which appeared circular in projection, consistent with spheres of average size $6.6 \pm 1.4 \mathrm{~nm}$ (Figure 1c). Conversely, Figure $1 \mathrm{~b}$ shows the same field of view, imaged at $850^{\circ} \mathrm{C}$, following rapid in situ heating (TP-1). In this case, the resultant HCNS structures were revealed, matching the positions of the starting TS-AgNP, whilst exhibiting a slightly larger average size of $8.9 \pm 1.7 \mathrm{~nm}$ (Figure 1d). Complementary EDS investigations (Figure 1e) confirmed the presence of Ag within the starting templated structures, whilst indicating its near complete removal from the HCNS product (See also SI-1a,b). 

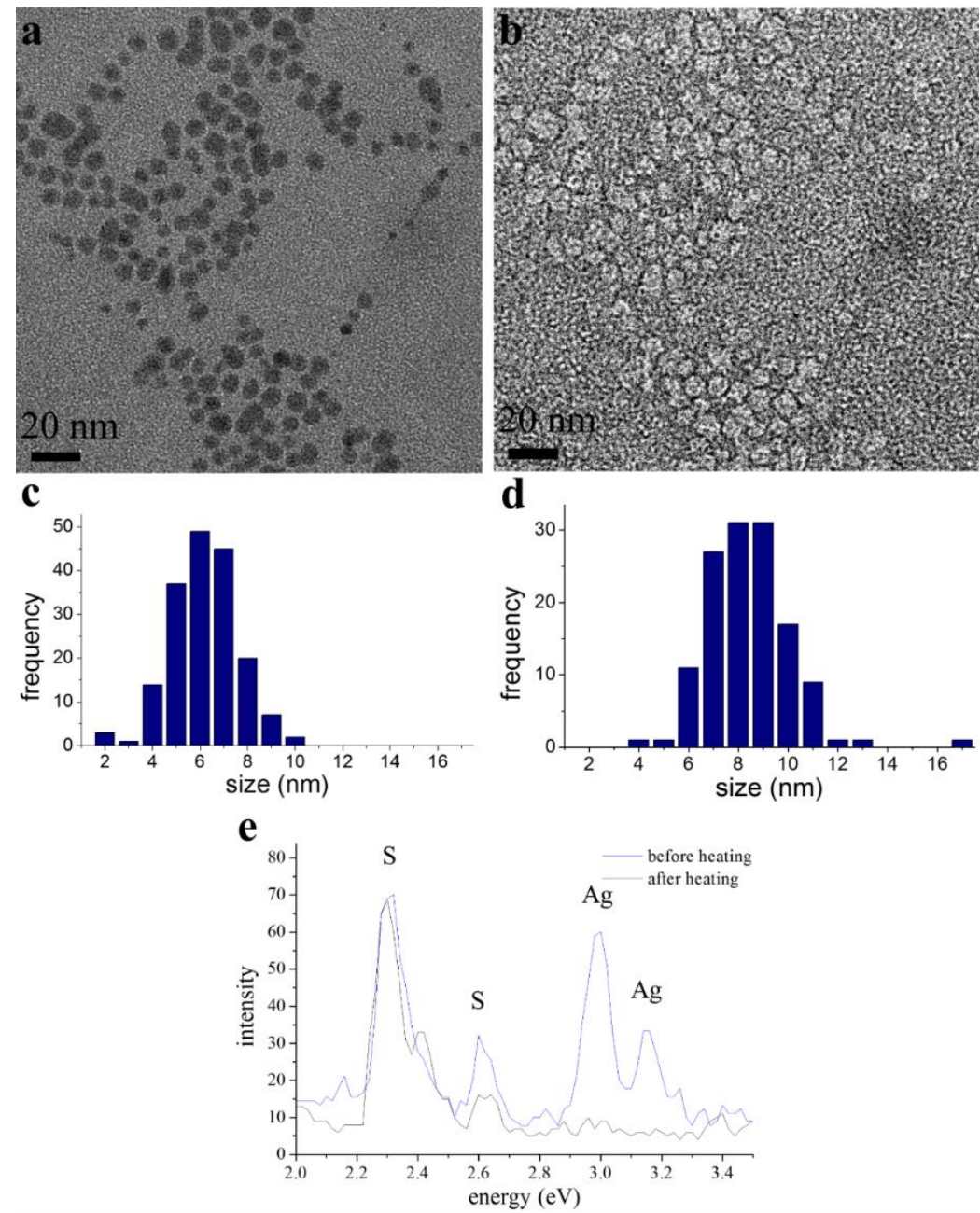

Figure 1. Bright field, diffraction contrast TEM micrographs of (a) TS-AgNP / $\mathrm{Si}_{3} \mathrm{~N}_{4}$ before rapid in situ heating to $850^{\circ} \mathrm{C}$ (TP-1); and (b) the resultant HCNS product, imaged at $850^{\circ} \mathrm{C}$ (scale bars $20 \mathrm{~nm}$ ). Particle size histograms: (c) before processing (corresponding to AgNP); and (d) after thermal processing (corresponding to HCNS). (e) Wide field of view EDS before and after thermal processing, indicating almost complete removal of $\mathrm{Ag}$ (See also SI-1a,b).

Figures 2a,b present high magnification, phase contrast TEM images of the developed HCNS, in this case recorded 8 days after processing, at RT, confirming the stability of the resultant structures. The majority of these particles, formed under TP-1 conditions, comprised complete, distinct carbon shells, being spherical in nature and hollow, consistent with the complete removal of the AgNP template. It is noted that a small proportion of these HCNS particles appeared to be fragmented (Figure 2a, arrowed), indicative of a heating rate effect on their formation. 

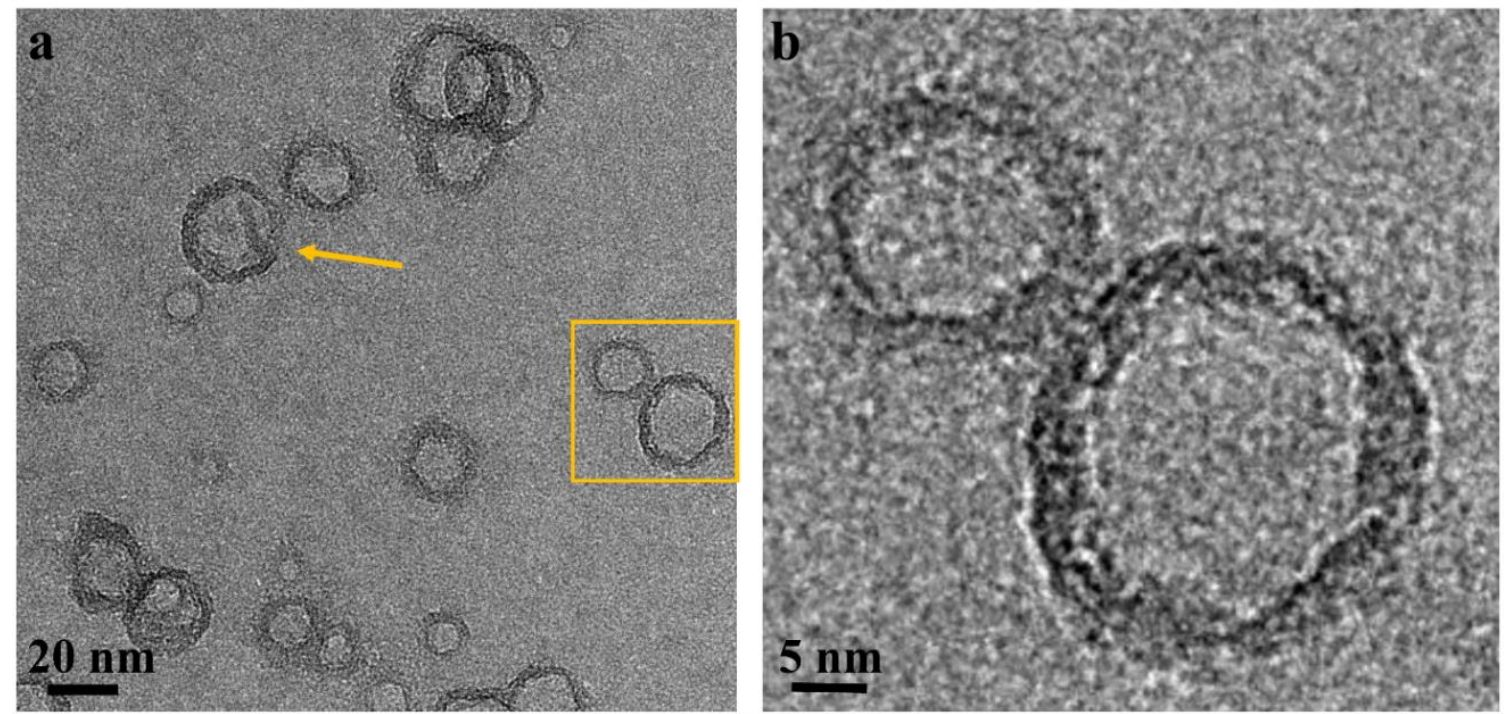

Figure 2. Phase contrast TEM micrographs providing a more detailed view of the resultant HCNS structures (TP-1; imaged at $100 \mathrm{kV}$ at RT). (a) Discrete HCNS and example fragmented HCNS (arrowed); and (b) High magnification image of region indicated by box in (a).

More detailed insights into the development of these HCNS structures were obtained through ex situ investigation, with furnace heating of TS-AgNP on MWCNT supports (TSAgNP@CNT) producing morphologies commensurate with the structures formed in situ. Figure 3a presents a low magnification, diffraction contrast TEM image of the starting TSAgNP decorated MWCNT, tilting of which allowed discrete NP to be imaged edge-on to the support (i.e. effectively free-standing). Figures 3b,c present higher magnification images of individual TS-AgNP and resultant HCNS, before and after ex situ heating at $850^{\circ} \mathrm{C}$ for $3 \mathrm{~h}$. Figure $3 \mathrm{~b}$ is illustrative of the amorphous thiol coated AgNP prior to thermal processing, whilst Figure $3 \mathrm{c}$ demonstrates unambiguously the hollow carbon shell structure. 

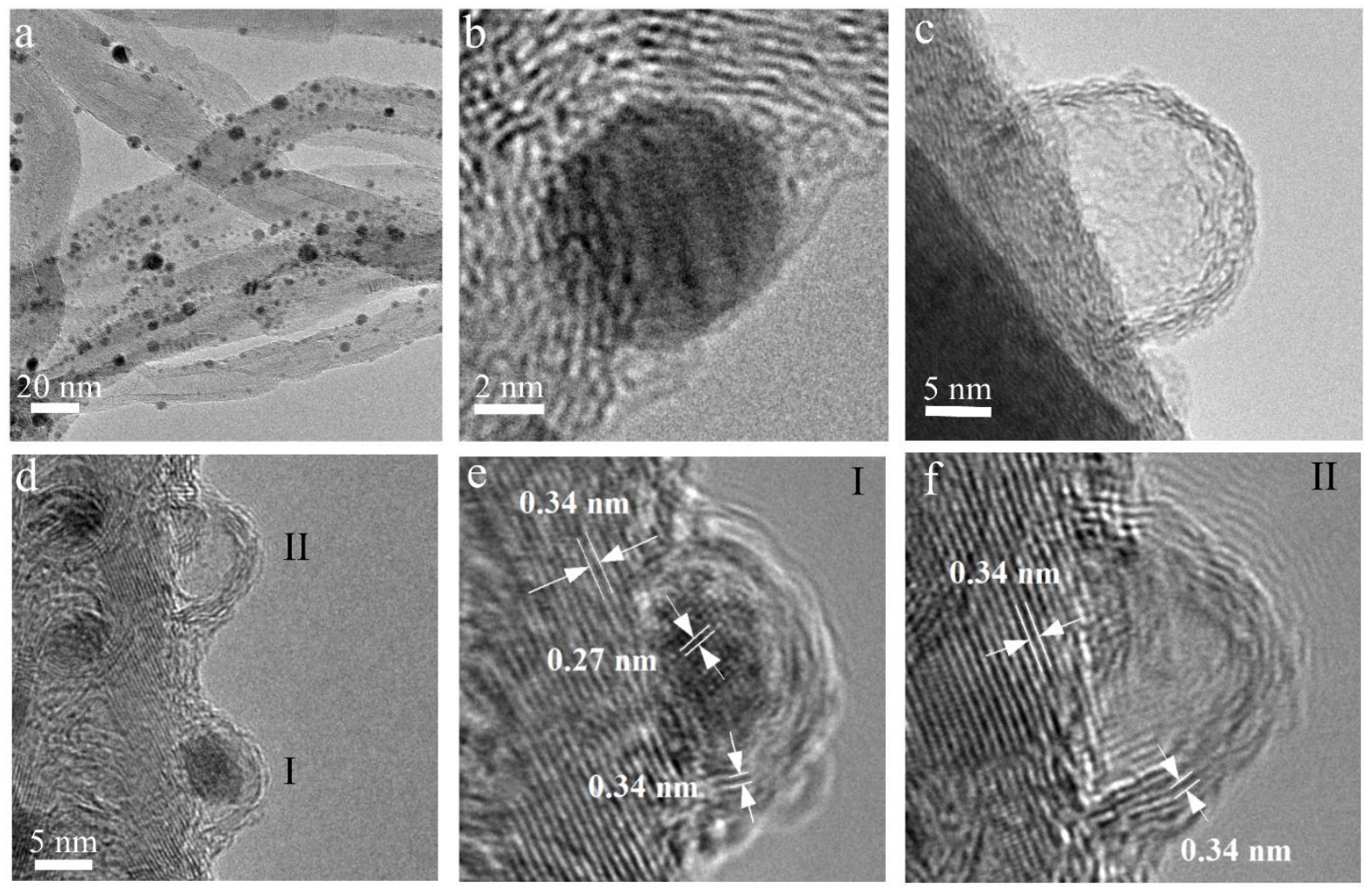

Figure 3. (a) Bright field diffraction contrast TEM micrograph of TS-AgNP@CNT and (b) higher magnification, TEM image of TS-AgNP@CNT, before ex situ heating at $850^{\circ} \mathrm{C}$ for $3 \mathrm{~h}$; (c) resultant HCNS, imaged at RT. (d) Phase contrast TEM image showing (e) Ag templated (l) and (f) hollow (II) carbon shell structures.

The TEM image of Figure 3d, recorded under flat-focus conditions, illustrates two neighbouring NP which happen to be (I) Ag-filled and (II) hollow. (A tilt series view of similar HCNS structures is presented in SI-2). The phase contrast image of Figure 3e, recorded under conditions of slight defocus, showed the development of an irregular, graphitic shell surrounding the $\operatorname{AgNP}$ (i.e. G-AgNP), whilst Figure $3 f$ confirmed the graphitic nature of the resultant hollow shell structure (G-HCNS). The HCNS walls comprised typically four layers, with graphitic spacing $0.34 \mathrm{~nm}$ [19]. It is noted that the walls of the resultant hollow carbon shell nanostructure form and graphitise at elevated temperature [20], prior to removal of the AgNP template, consistent with the catalytic activity of Ag during carbon shell formation [21]. The close proximity of templated G-AgNP and hollow G-HCNS in this instance is indicative of some variability in the activation for this transformation process.

Localised variability in the AgNP template removal was also observed depending on particle position on the support during in situ thermal processing, with fast heating rate to $850^{\circ} \mathrm{C}$ (TP1). Figure $4 \mathrm{a}$ shows a distribution of distinct $\mathrm{HCNS}$ on the $\mathrm{Si}_{3} \mathrm{~N}_{4}$ electron transparent windows 
of the MEMS chip, whilst an array of encapsulated CNS-AgNP decorate the edge of the support window. Further, it is noted that silver nanoparticles within the CNS-AgNP $(23.3 \pm 4.2 \mathrm{~nm}, \mathrm{n}$ $=6$ ), in this instance, were significantly larger than the starting TS-AgNP (Figure 1a), indicative that some AgNP Ostwald ripening had occurred [22]. These observations are again consistent with the suggestion that localised thermal effects mediate the onset and progression of this transformation process.
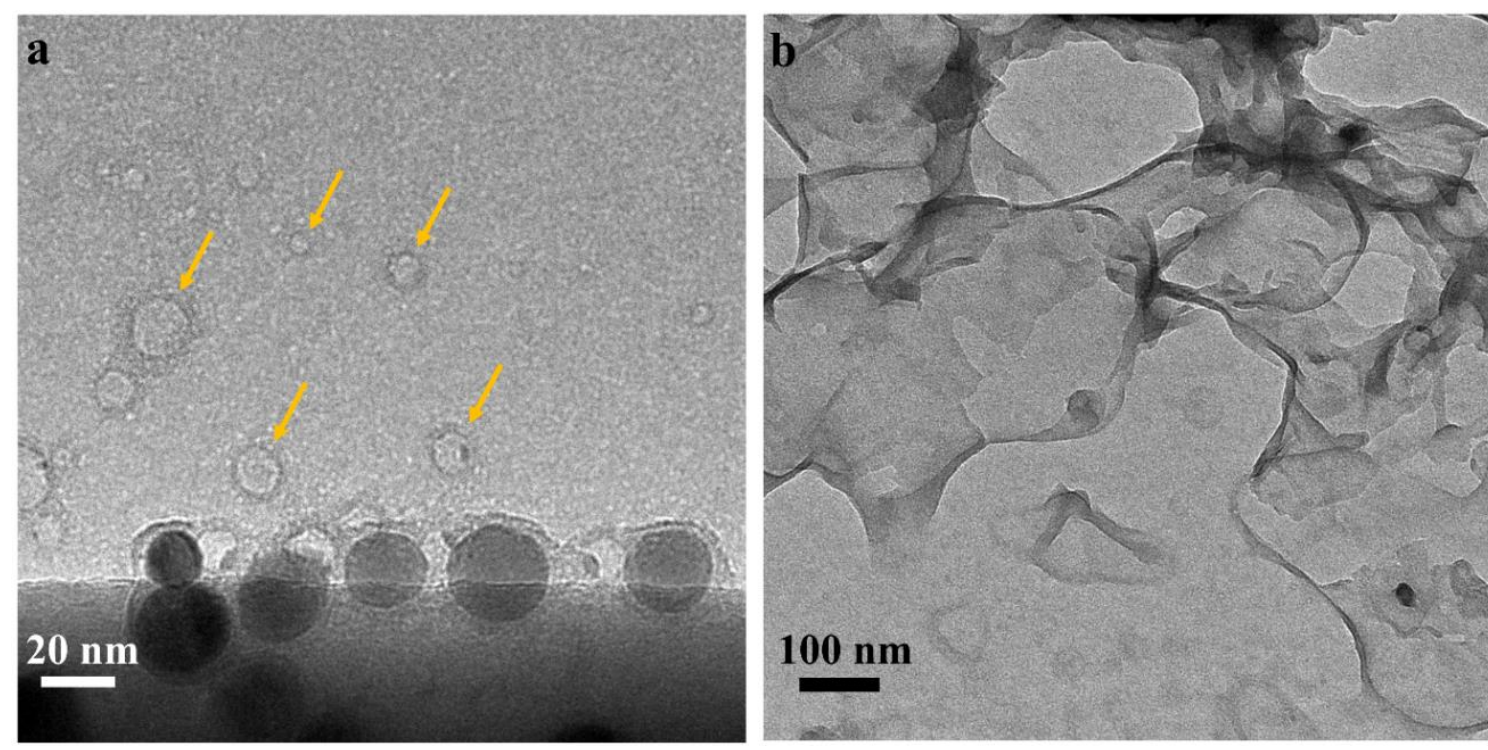

Figure 4. (a) Phase contrast TEM micrograph of HCNS indicated by arrows, adjacent to CNS-AgNP located on thicker regions of the $\mathrm{Si}_{3} \mathrm{~N}_{4}$ support, recorded at RT, following in situ TP-1; and (b), low magnification, bright field TEM image of lacy carbon structures formed during TP-2.

Figure $4 \mathrm{~b}$ presents an image of the lacy-carbon reaction product, formed from the same TSAgNP starting material, following in situ thermal processing with a slow heating rate up to $900^{\circ} \mathrm{C}$ (TP-2). In this case, there was a high degree of variability in the product across the centralised support windows of the MEMS heating chip, with a mixture of amorphous, lacy carbon interspersed with large accumulations of Ag (see also SI-3), along with some ill-defined HCNS structures. This demonstrates that heating rate is critical for the formation of welldefined HCNS structures. In particular, no small, discrete HCNS structures were evident.

Figure 5 presents first order Raman spectra acquired from the products formed from TS-AgNP thermally processed on $\mathrm{Si}_{3} \mathrm{~N}_{4}$ supports under conditions of fast (TP-1) and slow (TP-2) heating rates, respectively (See also SI and associated commentary). Both spectra are dominated by two broad, strongly overlapping features in the range $1000-1800 \mathrm{~cm}^{-1}$, nominally relating to 
the $\mathrm{D}$ (defect) band, i.e. an $\mathrm{A}_{1 \mathrm{~g}}$ breathing mode of six atom rings, requiring a defect for activation, along with the $\mathrm{G}$ (graphite) band, i.e. a carbon-carbon stretching vibration of $\mathrm{E}_{2 \mathrm{~g}}$ symmetry, present in all graphitic materials, but representing a superposition of at least five spectral contributions (see Table S1 in the Supporting Information for assignment and further discussion). Of note, the spectra are very similar to each other and consistent with that expected from a disordered graphitic nanocarbon. Conversely, complementary NMR and FTIR data demonstrated that the starting TS-AgNP materials were non-graphitic (SI-4 and SI-5, respectively).
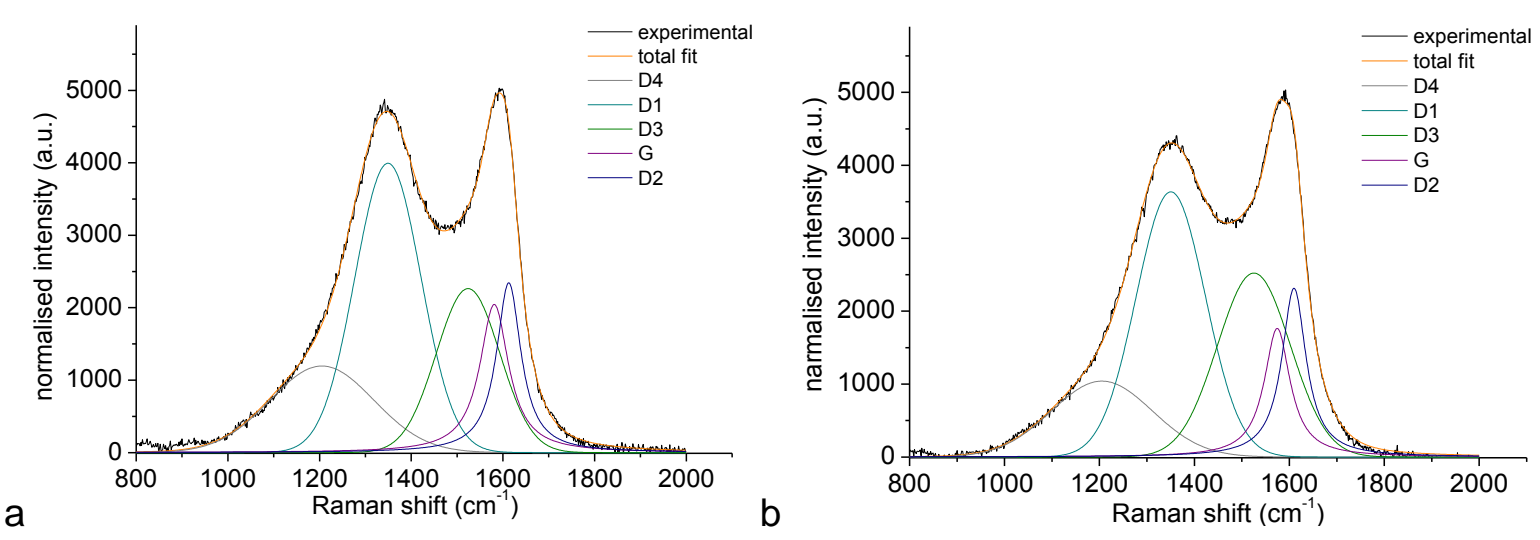

Figure 5. First order Raman spectra $\left(\lambda_{\mathrm{ex}}=532 \mathrm{~nm}\right)$ obtained in the range $800-2000 \mathrm{~cm}^{-1}$ from samples thermally processed using either: (a) fast (TP-1); or (b) slow (TP-2) heating rates, respectively, confirming the disordered graphitic nature of the products. Spectra have been normalised to the intensity of the $\mathrm{G}$ band, baseline-corrected using a third-order polynomial subtraction and fit using a five-band model analogous to that described previously [18] $\left(\chi^{2}=2.3\right.$ and 2.4 for (a) and (b), respectively).

\section{Discussion}

Discrete, $<10 \mathrm{~nm}$ sized HCNS have been formed from the single-step, thermal processing of TS-AgNP, by ex situ heating on a MWCNT support, or as observed in real time via in situ TEM on a $\mathrm{Si}_{3} \mathrm{~N}_{4}$ MEMS support. The identical location of the HCNS product with the starting dispersion of TS-AgNP, as observed during in situ TEM thermal processing (TP-1), provides direct evidence for the templated formation of HCNS on AgNP (Figure 1). In particular, the size of these HCNS particles $(8.9 \pm 1.7 \mathrm{~nm}$ for the sample set shown here) is defined by, and slightly larger than, the size of the AgNP template $(6.6 \pm 1.4 \mathrm{~nm})$, as compared with the previous smallest HCNS reported to date of $\sim 70 \mathrm{~nm}$ [23]. 
Most of the HCNS product, formed in situ under TP-1 processing conditions, comprised complete spheres, with the occasional observation of partially fragmented spheres (Figure 2), suggesting an effect of variable localised temperature, or rate of heating, on their formation. Nevertheless, the defining features for the product, i.e. hollow interior and $<10 \mathrm{~nm}$ size are clearly evident (Figure 3). For the case of structures observable free from background contributions at the edges of MWCNT supports (Figure 4), HCNS interlayer spacings commensurate with graphitic spacings $(0.34 \mathrm{~nm})$ [19] were observed, whilst noting that these structures are likely to be disordered [24] because of their very pronounced curvatures.

With regard to the HCNS products processed on planar $\mathrm{Si}_{3} \mathrm{~N}_{4}$ supports in situ (Figure 2), definitive visual evidence for graphitisation could not be obtained from phase contrast imaging, considered to be compromised by the morphology and thickness of the support, combined again with the very high levels of curvature associated with such $<10 \mathrm{~nm}$ sized shells, acting to mask the structural details of the developed shell walls. However, indirect evidence from complementary Raman spectroscopy confirmed the presence of highly disordered graphitic domains for samples processed in situ (Table SI-1; with high intensity peak ratios indicating the presence of both disordered and graphitic carbon $\left(\mathrm{I}_{\mathrm{D} 1}: \mathrm{I}_{\mathrm{G}}=3.08\right)$ [18]), suggesting that HCNS graphitisation at elevated temperature is not CNT template specific. Whilst a contribution from the support material on graphitisation cannot be discounted, it is considered that the process of graphitisation is thermally induced and catalysed by $\mathrm{Ag}$ [20] in this instance. Further, the ability to form commensurate HCNS structures by ex situ furnace heating of TSAgNP (Figure 4) confirms that this thermally mediated process is not governed by electron beam effects. Indeed, once formed, the HCNS appear to be very stable.

Accordingly, it is proposed that the formation of HCNS, from the thiol stabilising layer of TSAgNP, proceeds via thermally driven dehydrogenation [25], with the crosslinking of alkane chains, the transformation of $\mathrm{sp}^{3}$ to $\mathrm{sp}^{2}$ carbon during graphitisation, and finally Ag template removal (Figure 6). Indeed, the appearance of carbon shells surrounding AgNP (Figures 3d,e and Figure 4b), during both in situ and ex situ heating experiments, demonstrates that the development of carbon shells proceeds at the surfaces of AgNP, in advance of template removal. Hence, it is considered that the AgNP core acts both as a physical template for HCNS and catalyses the formation of carbon shells [26]. The residual presence of small amounts of $\mathrm{Ag}$ within some of the developed HCNS, indicative of incomplete processing, is also consistent with the removal of AgNP after shell formation. It is suggested that Ag migrates through the 
shells via structural defects [27]. With regard to the EDS observation of low S levels within the product, this heteroatom is known to terminate unsaturated vacancies at graphitic domain edges, resulting in thermodynamic stabilisation [28].
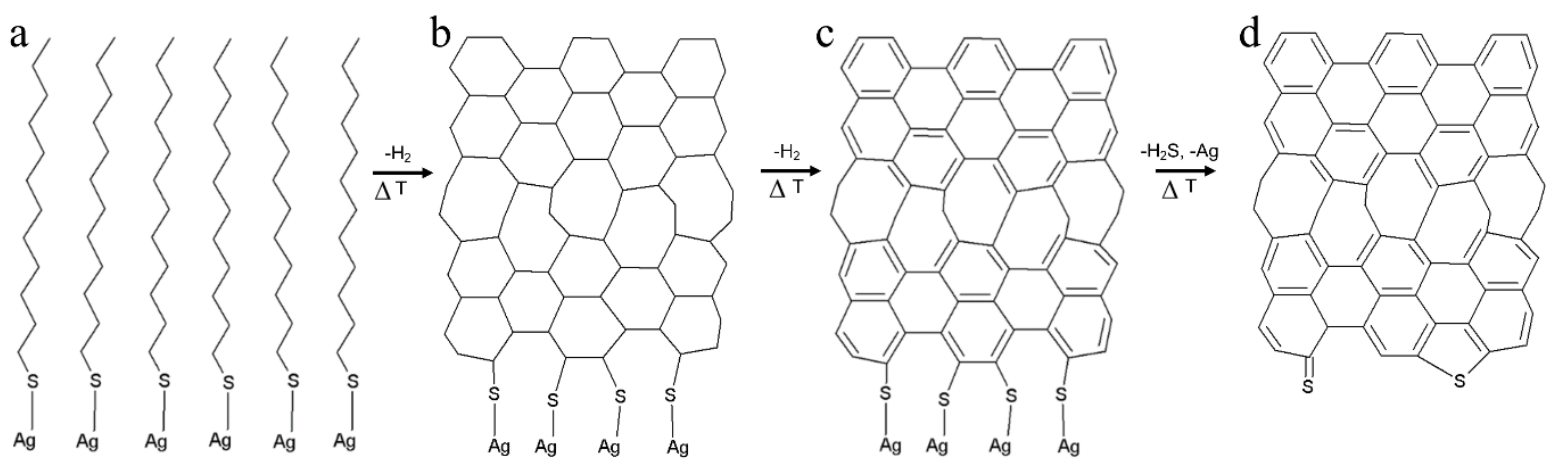

Figure 6. Proposed schematic mechanism for HCNS formation during rapid thermal processing (TP1), up to $850^{\circ} \mathrm{C}$ : (a) Surface of thiol stabilised AgNP; (b) cross-linking of alkane chains; (c) graphitisation; and (d) loss of AgNP template to leave a stabilised HCNS product.

Spatial variations in the nature of the HCNS product were evident for both in situ and ex situ processed samples, suggesting a combination of effects relating to individual NP size, temperature and heating rate. For example, the close proximity of filled and empty nanoshells processed ex situ (Figure 3) is considered indicative of a subtle NP size effect; whilst samples processed in situ under conditions of TP-1 showed examples of well-defined HCNS and regions of partially processed TS-AgNP, depending on position on the MEMS $\mathrm{Si}_{3} \mathrm{~N}_{4}$ chip, e.g. with larger encapsulated AgNP at the window edge (Figure 4), attributed to localised Ostwald ripening, indicative of a temperature (thermal conduction / thermal gradient) effect. Further, it is recognised that rate of heating is critical for the formation of well-defined HCNS. In particular, thermal processing of TS-AgNP under slow heating rate conditions (TP-2) in situ did not yield uniform HCNS. Hence, it is considered that the steady diffusion of Ag under slow heating rate conditions resulted in a degradation of the templating effect (with concomitant decrease in catalytic actvity and product graphitisation), in turn acting to disrupt the formation of spherical HCNS, leading the development of lacey, amorphous, and non-homogeneous carbon structures (Figure 4b), along with localised, large accumulations of Ag (SI 3). Indeed, complementary Raman spectroscopy demonstrated decreased levels of graphitic ordering within in situ slowly heated (TP-2) TS-AgNP ( $\mathrm{I}_{\mathrm{D} 1}: \mathrm{I}_{\mathrm{G}}=3.77$, consistent with increased disorder), as compared to samples processed under fast heating rate conditions (TP-1). For the 
purpose of materials control and the potential for scale-up, and from a wider reaction kinetics perspective, it is recognised that further work is needed to separate out the competing roles of temperature, heating rate, template size and support material on the development of these HCNS products.

The formation of bespoke HCNS (of sizes $>70 \mathrm{~nm}$ ) has been achieved to date via the use of multi-step processing, e.g. carbon coating of hard template materials, necessitating a synthetic step and thermal treatment, in advance of chemical template removal. Here, we have demonstrated that thiol stabilised AgNP, can be transformed into $<10 \mathrm{~nm}$ sized HCNS, using a facile one-step thermal process. This approach provides for excellent size control of the HCNS product via appropriate AgNP template selection.

\section{Conclusion}

Discrete, $<10 \mathrm{~nm}$ sized HCNS structures have been formed from TS-AgNP via heat induced transformation, using a facile one-step process, by either ex situ heating on a MWCNT support, or in situ heating within a TEM on a $\mathrm{Si}_{3} \mathrm{~N}_{4}$ MEMS support. Direct evidence for the templated formation of predominantly complete, spherical HCNS on AgNP under rapid heating conditions is presented, with the size of the resultant HCNS being slightly larger than that of the AgNP template. These very stable HCNS exhibited clearly the twin characteristics of hollow interiors and highly disordered, sphere wall graphitisation. It is proposed that the HCNS formation mechanism, from the thiol stabilising layer of TS-AgNP, proceeds via thermally driven dehydrogenation, with crosslinking of alkane chains and transformation of $\mathrm{sp}^{3}$ to $\mathrm{sp}^{2}$ during graphitisation, in advance of AgNP template removal. Accordingly, the AgNP core acts both as a physical template for HCNS and catalyses the graphitisation of carbon. Spatial variations in the development of the reaction product reflects predominantly a competition between the thermal transformation of the TS-layer and loss of the AgNP template effect. In particular, a fast heating rate is found to be critical for the formation of well-defined HCNS, whilst a slow heating rate gave a more variable product, comprising lacey, amorphous, and non-homogeneous carbon structures with decreased levels of graphitic ordering. It is considered that one-step thermal processing of thiol stabilised AgNP provides for excellent size control of the HCNS product, via appropriate AgNP template selection. 


\section{Acknowledgements}

The authors thank the Engineering and Physical Sciences Research Council (Award References EP/M506588/1 and EP/L022494/1) for support; and acknowledge Dr A. La Torre for training support and scientific discussions. With thanks also to Dr Emily F Smith at the Nanoscale and Microscale Research Centre (nmRC), University of Nottingham, for the acquisition of complementary XP spectra and associated data interpretation.

\section{References}

[1] V.V. Pokropivny, A.L. Ivanovskii, New nanoforms of carbon and boron nitride, Russ Chem Rev 77(10) (2008) 837 -873.

[2] M.J. Zhong, E.K. Kim, S. Natesakhawat, J.P. McGann, J.F. Whitacre, K. Matyjaszewski, et al., Nitrogen-rich porous nanocarbon from block copolymer precursors for $\mathrm{CO} 2$ capture and electrocatalysis of oxygen reduction, Abstr Pap Am Chem S 244 (2012).

[3] R. Blinc, D. Arcon, P. Umek, T. Apih, F. Milia, A.V. Rode, Carbon nanofoam as a potential hydrogen storage material, Phys Status Solidi B 244(11) (2007) 4308-4310.

[4] Q. Wang, X. Huang, Y. Long, X. Wang, H. Zhang, R. Zhu, et al., Hollow luminescent carbon dots for drug delivery, Carbon 59 (2013) 192-199.

[5] S. Azoubel, S. Magdassi, The formation of carbon nanotube dispersions by high pressure homogenization and their rapid characterization by analytical centrifuge, Carbon 48(12) (2010) 3346-3352.

[6] J.M. Cowley, C.-H. Kiang, The structure of near-spherical carbon nano-shells, Carbon 38 (1999) 1437-1444.

[7] Z. Sun, X. Song, P. Zhang, L. Gao, Template-assisted synthesis of multi-shelled carbon hollow spheres with an ultralarge pore volume as anode materials in Li-ion batteries, RSC Adv Vol.5(5) (2015) 3657-3664.

[8] N.M. Tun, A. N. Morozov., I.M. Izvol'skii., E.G. Rakov, Preparation and characterisation of hollow carbon nanospheres, Inorg Mater Vol.51(5) (2015) 445-450.

[9] Z.B. Lei, Z.W. Chen, X.S. Zhao, Growth of Polyaniline on Hollow Carbon Spheres for Enhancing Electrocapacitance, J Phys Chem C 114(46) (2010) 19867-19874.

[10] S. Yang, X. Feng, L. Zhi, Q. Cao, J. Maier, K. Müllen, Nanographene-Constructed Hollow Carbon Spheres and Their Favorable Electroactivity with Respect to Lithium Storage, Adv Mater 22(7) (2010) 838-842.

[11] P.M. Arnal, F. Schuth, F. Kleitz, A versatile method for the production of monodisperse spherical particles and hollow particles: Templating from binary core-shell structures, Chem Comm (11) (2006) 1203-1205.

[12] N.P. Wickramaratne, V.S. Perera, B.-W. Park, M. Gao, G.W. McGimpsey, S.D. Huang, et al., Graphitic Mesoporous Carbons with Embedded Prussian Blue-Derived Iron Oxide Nanoparticles Synthesized by Soft Templating and Low-Temperature Graphitization, Chem Mater 25(14) (2013) 2803-2811.

[13] F. Xu, Z. Tang, S. Huang, L. Chen, Y. Liang, W. Mai, et al., Facile synthesis of ultrahigh-surface-area hollow carbon nanospheres for enhanced adsorption and energy storage, Nature Commun 6 (2015) 7221. 
[14] N.A. Katcho, E. Urones-Garrote, D. Ávila-Brande, A. Gómez-Herrero, S. Urbonaite, S. Csillag, et al., Carbon hollow nanospheres from chlorination of ferrocene, Chem Mater 19(9) (2007) 2304-2309.

[15] X.Y. Lai, J.E. Halpert, D. Wang, Recent advances in micro-/nano-structured hollow spheres for energy applications: From simple to complex systems, Energy Environ Sci 5(12) (2012) 9944-9944.

[16] S.Y. Kang, K. Kim, Comparative study of dodecanethiol-derivatized silver nanoparticles prepared in one-phase and two-phase systems, Langmuir 14(1) (1998) 226-230.

[17] C.A. Schneider, W.S. Rasband, K.W. Eliceiri, NIH to ImageJ: 25 years of image analysis, Nat Methods 9(7) (2012) 671-5.

[18] A. Sadezky, H. Muckenhuber, H. Grothe, R. Niessner, U. Pöschl, Raman microspectroscopy of soot and related carbonaceous materials: Spectral analysis and structural information, Carbon 43(8) (2005) 1731-1742.

[19] S. Iijima, M. Yudasaka, R. Yamada, S. Bandow, K. Suenaga, F. Kokai, et al., Nanoaggregates of single-walled graphitic carbon nano-horns, Chem Phys Lett 309(3-4) (1999) 165-170.

[20] O. Endo, M. Nakamura, K. Amemiya, H. Ozaki, Graphene nanoribbons formed from nalkane by thermal dehydrogenation on $\mathrm{Au}(111)$ surface, Surf Sci (2015) 44-48.

[21] A. G. Ramirez, T. Itoh, R. Sinclair, Crystallization of amorphous carbon thin films in the presence of magnetic media J App Phy 85(3) (1999) 1508-1513.

[22] N.G. Bastus, J. Comenge, V. Puntes, Kinetically Controlled Seeded Growth Synthesis of Citrate-Stabilized Gold Nanoparticles of up to $200 \mathrm{~nm}$ : Size Focusing versus Ostwald Ripening, Langmuir 27(17) (2011) 11098-11105.

[23] F. Xu, Z.W. Tang, S.Q. Huang, L.Y. Chen, Y.R. Liang, W.C. Mai, et al., Facile synthesis of ultrahigh-surface-area hollow carbon nanospheres for enhanced adsorption and energy storage, Nat Commun 6 (2015) 7221 1-10.

[24] F. Banhart, The role of lattice defects in the formation of new carbon structures under electron irradiation, J Electron Microsc 51(suppl_1) (2002) S189-S194.

[25] J-J. Shih, K-H. Kuo, C-M Yang, J-L. Lin, L-J. Fan, Y-W. Yang, Thermal dissociation of $\mathrm{HSCH}_{2} \mathrm{CH}_{2} \mathrm{OH}$ on $\mathrm{Cu}(111$ ). Surf Sci 602(21) (2008) 3266-3271.

[26] Y. Yaakob, M. Z. M. Yusop, C. Takahashi, M. S. Rosmi, G. Kalitaa, M. Tanemura, In situ transmission electron microscopy of Ag-incorporated carbon nanofibers: the effect of $\mathrm{Ag}$ nanoparticle size on graphene formation, RSC Adv 8 (5) (2015) 5647-5651.

[27] F. Banhart, P. Redlich, P.M. Ajayan, The migration of metal atoms through carbon onions, Chem Phys Lett 292(4) (1998) 554-560.

[28] A. Chuvilin, E. Bichoutskaia, M. C. Gimenez-Lopez, T. W. Chamberlain, G. A. Rance, N. Kuganathan, et al., Self-assembly of a sulphur-terminated graphene nanoribbon within a singlewalled carbon nanotube. Nat Mater 10(9) (2011) 687-692.

[29] L.G. Cançado, A. Jorio, E.H.M. Ferreira, F. Stavale, C.A. Achete, R.B. Capaz, et al., Quantifying defects in graphene via Raman spectroscopy at different excitation energies, Nano lett 11(8) (2011) 3190-6.

[30] A. Ferrari, J. Robertson, Interpretation of Raman spectra of disordered and amorphous carbon, Phys Rev B 61(20) (2000) 14095-14107.

[31] M. Hasan, D. Bethell, M. Brust, The Fate of Sulfur-Bound Hydrogen on Formation of Self-Assembled Thiol Monolayers on Gold: 1H NMR Spectroscopic Evidence from Solutions of Gold Clusters, J Am Chem Soc (2002) 124 (7), pp 1132-1133. 
[32] M. Brust, M. Walker, D. Bethell, D. J. Schiffrin, R. J. Whyman, Synthesis of thiolderivatised gold nanoparticles in a two-phase Liquid-Liquid system, Chem Soc Chem Commun (1994) 0 801-802.

[33] M. J. Hostetler, J. J. Stokes, R. W. Murray, Infrared spectroscopy of three dimensional self-assembled monolayers: $\mathrm{N}$-alkanethiolate monolayers on gold cluster compounds, Langmuir (1996) 12 3604-3612. 


\section{Supplementary Information}

\section{Electron Microscopy}
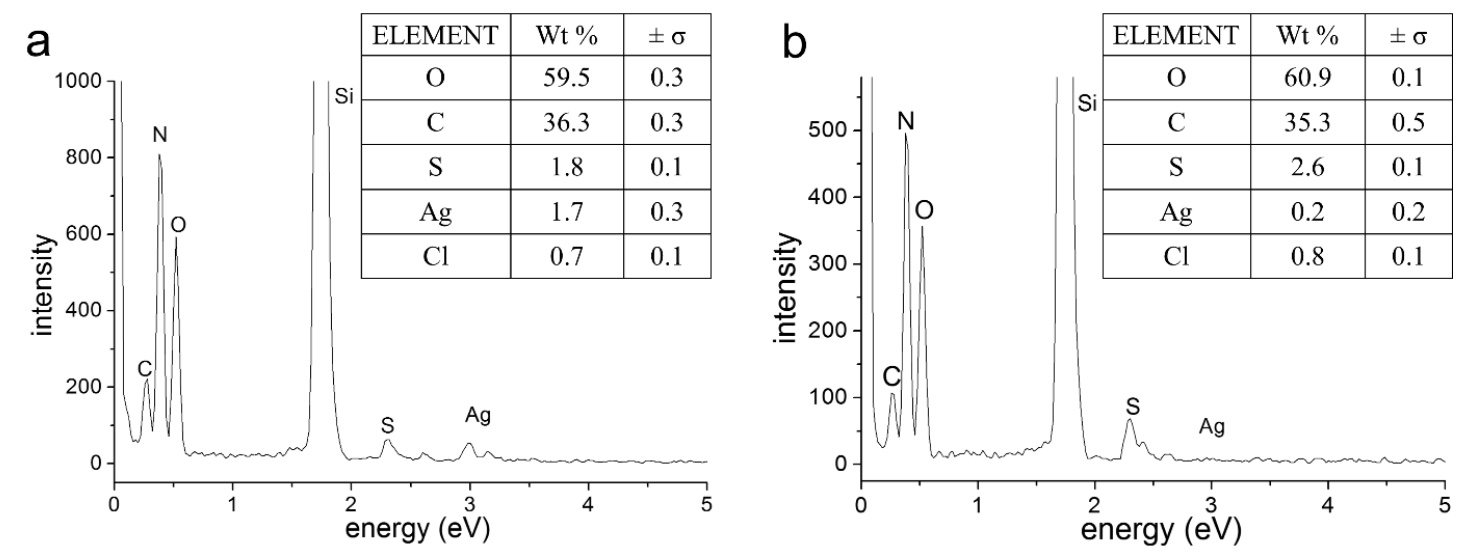

SI Figure 1 EDS spectra (recorded at RT) for TS-AgNP dispersed onto a planar $\mathrm{Si}_{3} \mathrm{~N}_{4}$ support: (a) before; and (b) after thermal heating to $850^{\circ} \mathrm{C}$ (TP-1), demonstrating near complete removal of $\mathrm{Ag}$ from the carbon shell product.
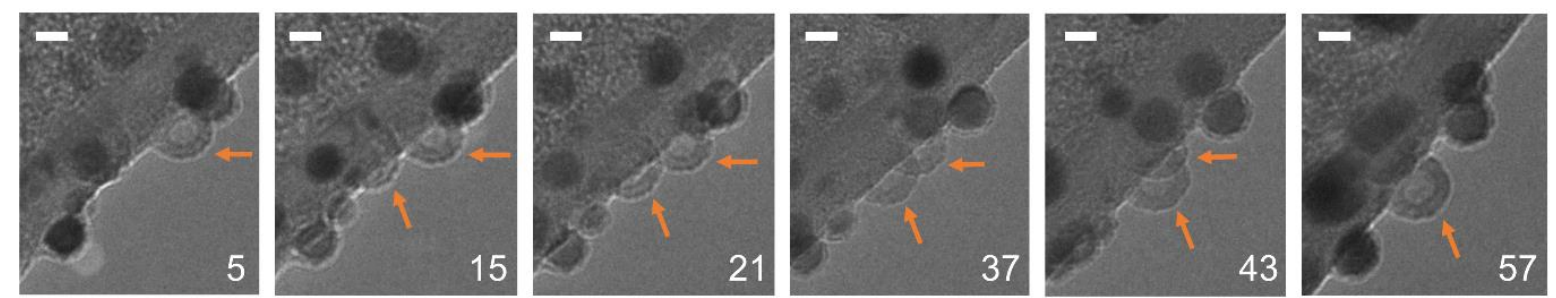

SI Figure 2 Complementary TEM images from a tilt series of HCNS, produced from TS-AgNP on $\mathrm{CNT}$, thermally processed ex situ at $850^{\circ} \mathrm{C}$ for $3 \mathrm{~h}$ (TP-1), acquired using a JEOL $2100 \mathrm{~F}$ operating at $100 \mathrm{kV}$; tilt angle in degrees; scale bar $10 \mathrm{~nm}$. Such data sets confirmed the hollow, spherical nature of these products and their graphitisation at elevated temperature when viewed at higher magnification (Figure 3f), along with the close proximity of filled and hollow shells ( $c f$. Figure 3d).
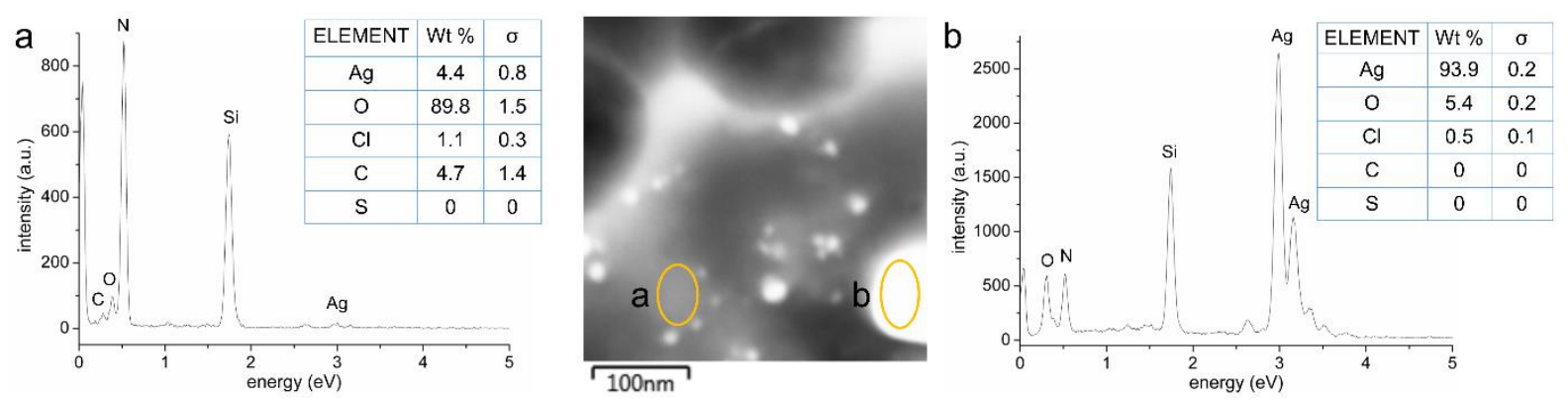

SI Figure 3 Dark field STEM and associated EDS data from regions a \& b, showing localised, large accumulations of Ag following thermal processing of TS-AgNP under a slow heating rate (TP-2). JEOL 2100 Plus operating at $80 \mathrm{kV}$. 


\section{Raman Spectroscopy}

Table SI-1. First order Raman bands assignment [18, SI-1, SI-2] (vs = very strong, s = strong, $\mathrm{m}=$ medium, $\mathrm{w}=$ weak).

\begin{tabular}{|c|c|c|c|}
\hline \multirow{2}{*}{ Band $^{\mathbf{a}}$} & \multicolumn{2}{|c|}{ Raman shift $/ \mathbf{c m}^{-\mathbf{1}}$} & \multirow{2}{*}{ Proposed vibrational mode assignment } \\
\cline { 2 - 3 } & TP-1 & $\mathbf{T P - 2}$ & \\
\hline G & $1581 \mathrm{~s}$ & $1574 \mathrm{~s}$ & Ideal graphitic lattice $\left(\mathrm{E}_{2 \mathrm{~g}}\right.$ symmetry) \\
\hline D1 (D) & $1349 \mathrm{vs}$ & $1350 \mathrm{vs}$ & Disordered graphitic lattice (A A $_{1 \mathrm{~g}}$ symmetry) \\
\hline D2 (D') & $1613 \mathrm{~s}$ & $1610 \mathrm{~s}$ & Disordered graphitic lattice (E $\mathrm{E}_{2 \mathrm{~g}}$ symmetry) \\
\hline D3 (D”, A) & $1524 \mathrm{~m}$ & $1525 \mathrm{~m}$ & Amorphous carbon \\
\hline D4 (I) & $1205 \mathrm{~m}$ & $1205 \mathrm{~m}$ & $\begin{array}{c}\text { Disordered graphitic lattice }\left(\mathrm{A}_{1 \mathrm{~g}} \text { symmetry), }\right. \\
\text { polyenes, ionic impurities }\end{array}$ \\
\hline
\end{tabular}

a alternative band designations of earlier studies are in parantheses

Subtle differences in the first order Raman spectra are noted for carbon shells prepared in situ using TP-1 and TP-2, including a shift in the position of the apparent G band from $~ 1595$ to $\sim 1590 \mathrm{~cm}^{-1}$, respectively. The difference in the position of this diagnostic band can be tentatively rationalised by either: (a) The presence of residual $\mathrm{Ag}$ in the carbon structure afforded, using a slow heating rate, resulting in a red shifted $\mathrm{G}$ band due to either plasmonic heating or charge transfer effects; or (b) differences in the effective curvature of the nanostructures obtained. The general peak shapes in the first order spectra (Figure 5) and weak intensity features in second order spectra associated with combination modes and overtones (data not shown) are both consistent with the formation of highly disordered graphitic carbon.

Also noted are differences in the relative intensities of the apparent D and $\mathrm{G}$ bands. Whilst the intensity ratio of $D$ and $G$ bands $\left(\mathrm{I}_{\mathrm{D}}: \mathrm{I}_{\mathrm{G}}\right)$ is often used to quantify disorder in graphitic nanostructures, its direct application to more disordered graphitic structures, such as those obtained here, is often unreliable. This relates to the apparent ambiguity associated with the use of peak heights or areas to define $\mathrm{I}_{\mathrm{D}}: \mathrm{I}_{\mathrm{G}}[\mathrm{SI}-1]$, the non-linearity in the relationship between $\mathrm{I}_{\mathrm{D}}: \mathrm{I}_{\mathrm{G}}$ and inter-defect distance within the graphitic lattice itself [SI-2], and the understanding that the region between $1000-1800 \mathrm{~cm}^{-1}$ more likely represents a superposition of at least five different spectral contributions, including D and G bands (SI-Table 1), which require careful deconvolution for comparative analysis.

Accordingly, fitting of the spectra obtained here and subsequent evaluation of the associated peak areas yielded some interesting quantitative information into the extent of disorder and amorphous $\mathrm{C}$ in the HCNS. The intensity ratio (as peak area) of the D1 and G bands $\left(\mathrm{I}_{\mathrm{D} 1}: \mathrm{I}_{\mathrm{G}}\right)-$ a measure of the disorder/order in the graphitic domains - is high, and greater for the carbon 
obtained with a slower heating rate, TP-2 ( $\mathrm{I}_{\mathrm{D} 1}: \mathrm{I}_{\mathrm{G}}=3.08$ and 3.77 for TP-1 and TP-2, respectively). The intensity ratio (as peak area) of the $\mathrm{D} 3$ and $\mathrm{G}$ bands $\left(\mathrm{I}_{\mathrm{D} 3}: \mathrm{I}_{\mathrm{G}}\right)$ - a metric used to quantity the ratio of amorphous carbon to graphitic domains - is also high and significantly higher for the shells obtained with a slower heating rate $\left(\mathrm{I}_{\mathrm{D} 3}: \mathrm{I}_{\mathrm{G}}=1.68\right.$ and 2.76 for TP-1 and TP-2, respectively).

\section{Nuclear Magnetic Resonance Spectroscopy}

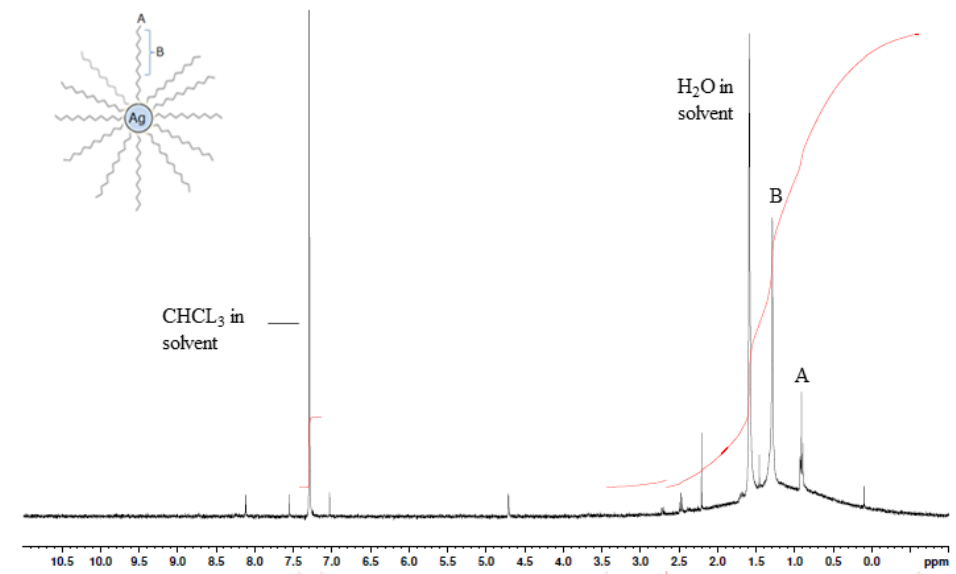

SI Figure $4{ }^{1} \mathrm{H}$ NMR spectrum and peak assignments for DDM stabilised AgNP, being consistent with literature examples [SI-3].

Evidence for the presence of DDM stabilised AgNP (TS-AgNP) and the absence of aromatic carbon groups is provided by NMR spectroscopy data (Figure SI-4a), being consistent with that of literature examples of DDM stabilised AgNP [SI-3], with peaks at $0.9 \mathrm{ppm}$ and in the range $1.2-1.6 \mathrm{ppm}$ assigned to the alkane DDM chain. No peaks are evident in the region associated with aromatic functional groups. Hence, the starting TS-AgNP is non-graphitic.

\section{Infrared Spectroscopy}

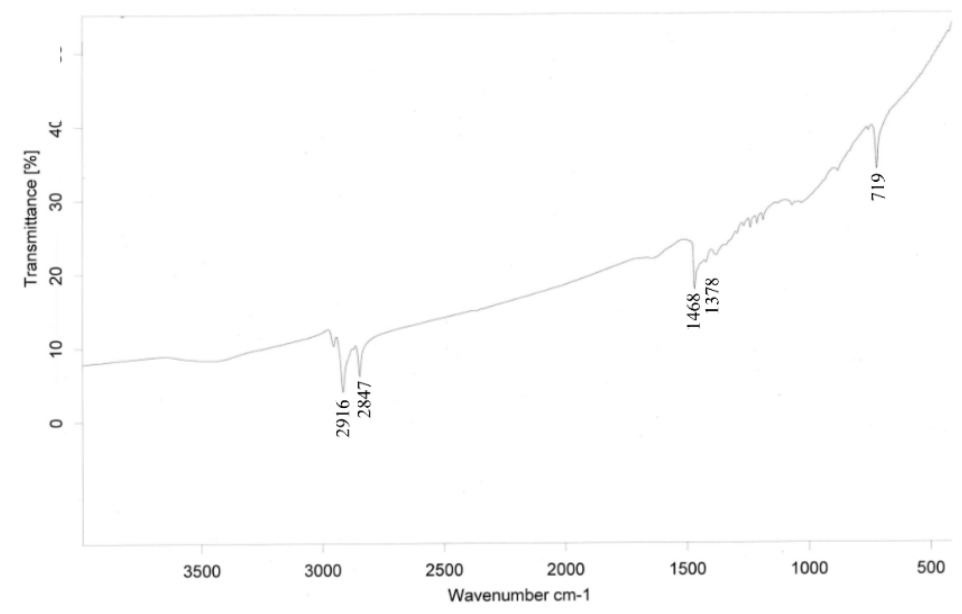


SI Figure 5 FTIR spectrum for TS-AgNP dispersed in $\mathrm{KBr}$, in agreement with published data of DDM stabilised AgNP.

Alkane $\mathrm{CH}_{2}$ symmetric and asymmetric stretching bands usually observed in DDM IR spectra at 2853 and $2924 \mathrm{~cm}^{-1}$, respectively, are shifted slightly to 2847 and $2916 \mathrm{~cm}^{-1}$, as observed in published spectra for DDM stabilised AgNP [SI-4], whilst the expected twisting-rocking and wagging bands of DDM in the 1150-1400 $\mathrm{cm}^{-1}$ region are observed at 1378 and $1468 \mathrm{~cm}^{-1}$, respectively; the latter $\mathrm{CH}_{2}$ scissoring band position is typical of all trans zig-zag chains of DDM stabilising AgNP [SI-5]. Peaks due to aromatic carbon functional groups around 1600 $\mathrm{cm}^{-1}$ are not observed. Hence, the starting TS-AgNP is non-graphitic.

\section{References}

[SI-1] L.G. Cançado, A. Jorio, E.H.M. Ferreira, F. Stavale, C.A. Achete, R.B. Capaz, et al., Quantifying defects in graphene via Raman spectroscopy at different excitation energies, Nano. Lett. 11(8) (2011) 3190-6.

[SI-2] A. Ferrari, J. Robertson, Interpretation of Raman spectra of disordered and amorphous carbon, Phys. Rev. B 61(20) (2000) 14095-14107.

[SI-3] M. Hasan, D. Bethell, M. Brust, The Fate of Sulfur-Bound Hydrogen on Formation of Self-Assembled Thiol Monolayers on Gold: 1H NMR Spectroscopic Evidence from Solutions of Gold Clusters, J Am. Chem. Soc. (2002) 124 (7), pp 1132-1133.

[SI-4] M. Brust, M. Walker, D. Bethell, D. J. Schiffrin, R. J. Whyman, Synthesis of thiolderivatised gold nanoparticles in a two-phase Liquid-Liquid system, Chem. Soc. Chem. Commun. (1994) 0 801-802.

[SI-5] M. J. Hostetler, J. J. Stokes, R. W. Murray, Infrared spectroscopy of three dimensional self-assembled monolayers: $N$-alkanethiolate monolayers on gold cluster compounds, Langmuir (1996) 12 3604-3612. 

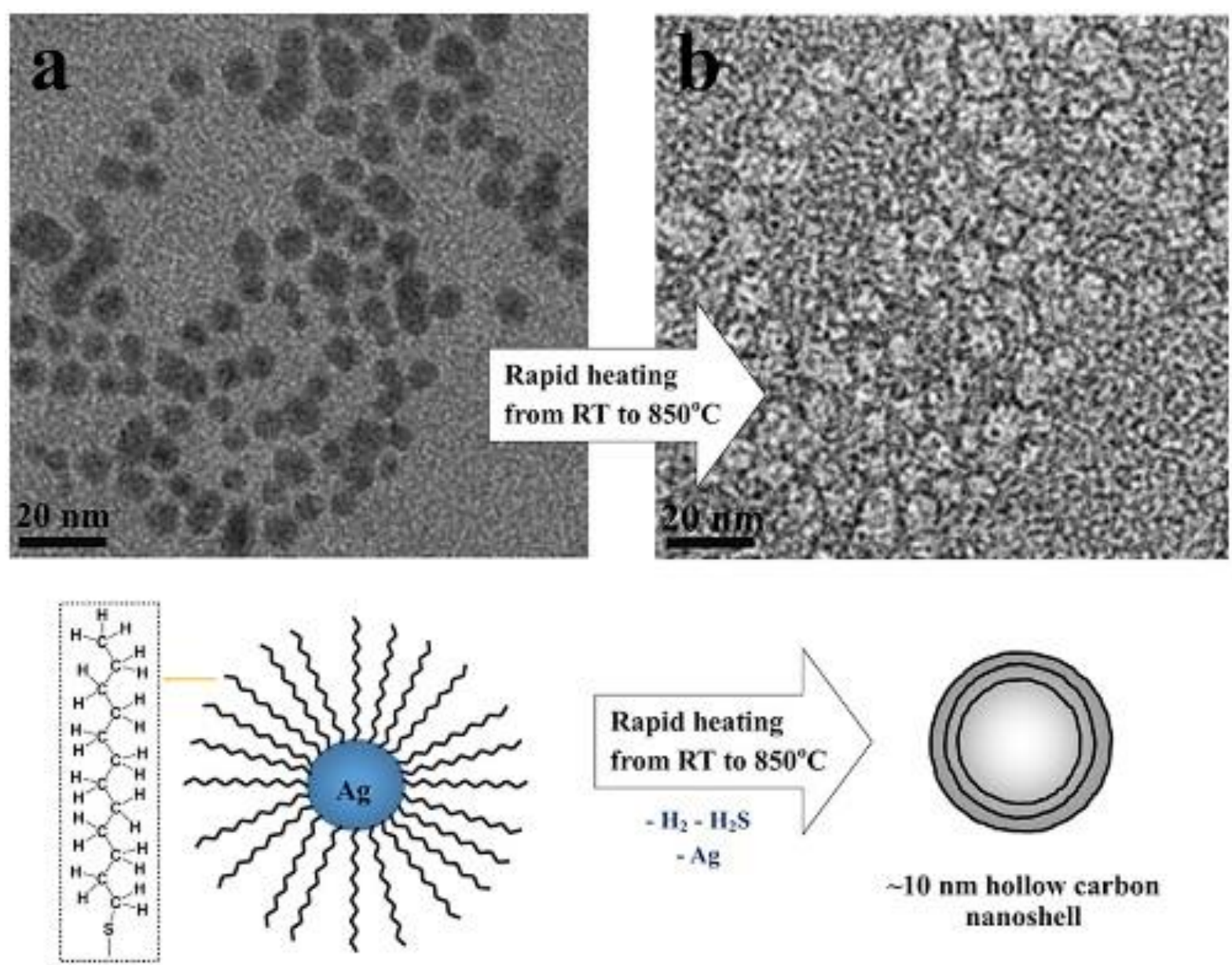